\title{
Frequency-Selective Beamforming Array Antenna Systems with Frequency-Dependent Phase Shifters
}

\author{
Jung Ouk Kim ${ }^{1} \cdot$ Won-Sang Yoon ${ }^{2} \cdot$ Sang-Min Han ${ }^{1, *}$
}

\begin{abstract}
A frequency-selective beamforming array antenna system is proposed for next-generation mobile applications. Whereas a conventional phased array steers antenna beams in the same direction, the proposed system involves a channel-selective beam for each frequency channel using RF/analog processing. A frequency-dependent phase shifter is designed with controllable phase slopes using reconfigurable transmission line configuration. Wideband frequency-dependent phase bits of $0^{\circ}, \pm 90^{\circ}, \pm 120^{\circ}, \pm 180^{\circ}, \pm 240^{\circ}$, and $\pm 270^{\circ}$ phase differences are achieved for a frequency band from $3.4 \mathrm{GHz}$ to $3.7 \mathrm{GHz}$. A wideband planar antenna element with a dual-finger monopole and an 8-way wideband matched feedline are applied to the proposed system. From the experimental results, the frequency-selective beamforming array presents simultaneous channel-selective beams with steering angles of up to about $\pm 40^{\circ}$. The adjustable beamforming system can achieve inter-beam hand-off for $5 \mathrm{G}$ mobile applications.
\end{abstract}

Key Words: Beamforming Antennas, Beam Steering, Phase Control, Phase Shifters, RF/Analog Processing.

\section{INTRODUCTION}

Since the system requirements for the fifth-generation (5G) mobile communication were released, related research has rapidly accelerated toward implementing core technologies such as millimeter waves, micro cells, massive multiple-input multipleoutput (MIMO), full duplexing, and adaptive beamforming [1, 2]. Although the 3GPP LTE Release 16 is expected to be the final specification of the $5 \mathrm{G}$ system to be released at the end of 2019, some leading companies have provided commercial $5 \mathrm{G}$ services since early 2019.

To achieve high-speed and real-time services, the 5G system adopted a broad bandwidth at a millimeter wave band with high propagation loss. To overcome the demerits, the system is orga- nized with adaptive beamforming and massive MIMO systems. These technologies require a numbers of antenna elements, complicated beamforming networks, and phase control circuits for each channel. For the accurate control of adaptive beams, recent novel approaches have been introduced, including phased arrays in millimeter-waves [3], switchable beamforming networks [4], beamforming on cellphones [5], and digital/hybrid beamforming [6]. In addition, a wideband antenna element is required to cover the ultra-wideband $[7,8]$, while the agile phase control circuits with high phase resolution should be considered to achieve wideband characteristics [9-11]. Moreover, wideband component design is an important issue regarding the 'sub-6 GHz band' to make the same beam shape for each channel. The only solution adjusting antenna beams for each channel is digital beamforming. Due to their high power-consumption

Manuscript received April 16, 2019 ; Revised May 31, 2019 ; Accepted August 8, 2019. (ID No. 20190416-020J)

${ }^{1}$ Department of Information and Communication Engineering, Soonchunhyang University, Asan, Korea.

${ }^{2}$ Division of Electronics and Display Engineering, Hoseo University, Asan, Korea.

"Corresponding Author: Sang-Min Han (e-mail: auspice@ieee.org)

This is an Open-Access article distributed under the terms of the Creative Commons Attribution Non-Commercial License (http://creativecommons.org/licenses/by-nc/4.0) which permits unrestricted non-commercial use, distribution, and reproduction in any medium, provided the original work is properly cited.

(c) Copyright The Korean Institute of Electromagnetic Engineering and Science. 
and expensive digital transmitters needed for each chain, digital beamforming is inefficient design for mobile terminals.

In this paper, a channel-selective beam controlled phased array antenna system is proposed under $\mathrm{RF} / \mathrm{analog}$ processing for the sub- $6 \mathrm{GHz}$ band. The phase control circuit was designed with different phase velocities for each antenna element. Due to the different phase steps between elements at each frequency channel, each channel beam steers in different directions. By adjusting the slopes of the phase characteristics within the phase control circuits, each channel beam can be steered in another direction. This paper is organized as follows. After a brief introduction, the frequency-selective phased array antenna system is described in Section II, whereas wideband components including frequency-dependent phase shifters are designed in Section III. Section IV presents the implementation and performance evaluation of the proposed system. The feasibility and applicability of the proposed system are discussed in the conclusion.

\section{FREQUENCY-SELECTIVE BEAMFORMING ARRAY}

\section{ANTENNA SYSTEM}

While a conventional analog phased array makes beams steer in the same direction for all frequency channels, the $5 \mathrm{G}$ system requires selective beam direction for each channel with digitally controlled beamforming, as shown in Fig. 1. While the conventional array steers the beams for all channels, the proposed array radiates a channel-selective beam for each channel in a simultaneous manner, like digital beamforming.

In this paper, an analog frequency-selective phase shifter was applied to each element with controllable phase slopes, as shown in Fig. 2(a). While the same phase is maintained at a center frequency channel, the left-to-right phase shift steps between the antenna elements increase at lower frequency channels and decrease at higher channels. Therefore, the proposed frequency-selective array can radiate uniform beam directions for all channels, in case of the same phase bits for eight elements as shown in Fig. 2(b). When the frequency-dependent phase shifters switch their phase bits in $+90^{\circ}$ increments at $3.4 \mathrm{GHz}$, $-90^{\circ}$ decrements at $3.7 \mathrm{GHz}$, and $0^{\circ}$ at $3.55 \mathrm{GHz}$, respectively, the radiation beams steer at separate angles, as shown in Fig. 2(c). As the phase steps change to $\pm 120^{\circ}$, the separating beam angles increase; therefore, the proposed channel-selective beamforming system can track moving users and make it possible to conduct an inter-beam hand-off, which subsequently makes the network system efficient in terms of its $5 \mathrm{G}$ mobile applications.

The proposed beamforming system was designed at a sub- 6 $\mathrm{GHz}$ band of 3.42-3.7 GHz for the $5 \mathrm{G}$ frequency band. Using the frequency-dependent phase shifter, the antenna beams at each channel simultaneously radiated in different directions. An

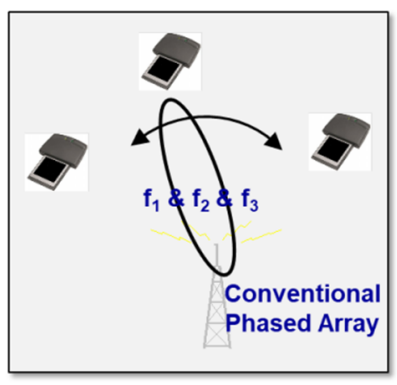

(a)

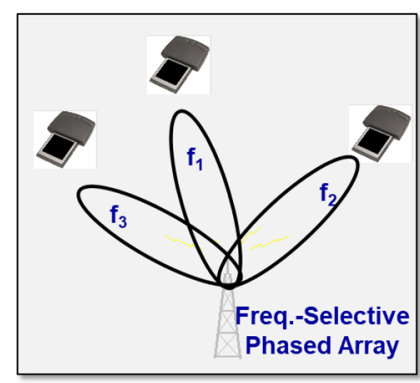

(b)
Fig. 1. Comparison of (a) conventional array antenna systems and (b) proposed frequency-selective beamforming.

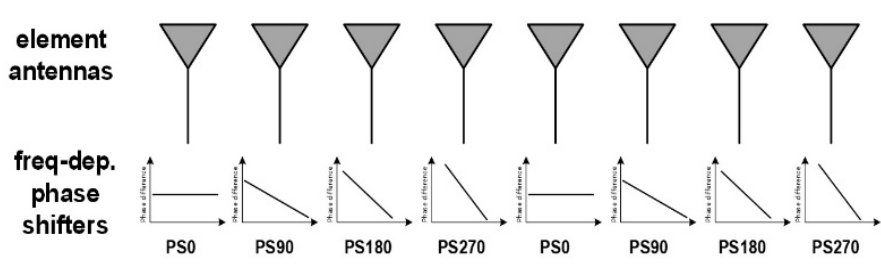

(a)

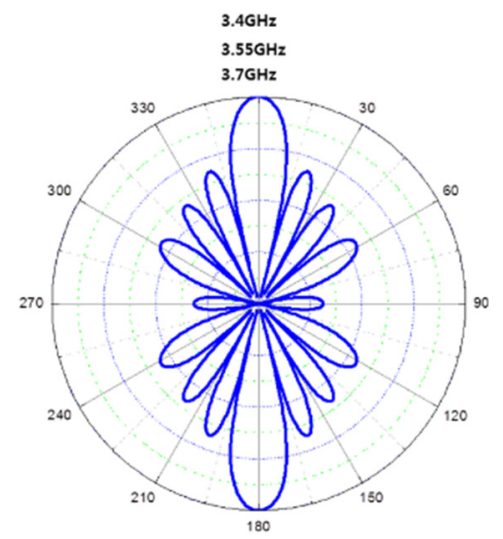

(b)

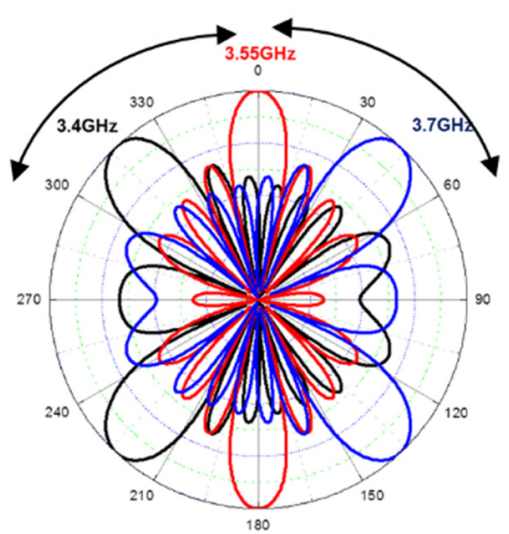

(c)

Fig. 2. Concept of the frequency-selective array antenna system: (a) phase characteristics of each antenna element, ideal radiation pattern, (b) with the same phase bits, and (c) with frequency-dependent phase bits.

$8 \times 1$ array was configured of planar dual-finger monopole elements, connecting frequency-dependent phase shifters with an 
8-way equal power divider circuit.

\section{FREQUENCY-SELECTIVE BEAMFORMING CIRCUIT DESIGNS}

\section{Frequency-Dependent Phase Shifter Design with Controllable Phase Slopes}

To make different phase steps for each frequency channel, the frequency-selective phase shifter was designed with an adjustable phase slope for each phase shift bit. Although the adjustable phase slope can be realized through tunable resonant circuits, it is limited by its narrow bandwidth; therefore, because the phase shifter with the most linear phase in the wide frequency band is a transmission line with a reconfigurable length and the phase velocity is dependent on the length of the transmission line, the frequency-dependent phase shifter with controllable phase slopes was designed in a meander configuration, as shown in Fig. 3. The extended transmission line can cause different time delays for each antenna element, which generates radiation beam squinting for wideband channel systems. Due to its wider channel bandwidth, the radiation beam can steer in more directions at both channel edge frequencies; therefore, the presented delay line phase shifter could be applied to the limited channel bandwidth. Additionally, since PIN diodes were mounted between the meander lines, current path lengths could be controlled with ON/OFF switching.

The proposed phase shift is controlled by the length of the microstrip line. Fig. 4(a) shows the shortest line length of a straight line corresponding to the slowest phase speed, while the fastest phase shift corresponding to the longest line length in Fig. 4(b). By switching the PIN diodes, the path length adjusts. As a phase bit is a relative value, the slowest phase slope is set to a reference value (PSO). All phase bits have $0^{\circ}$ phase shifts at a center frequency of $3.55 \mathrm{GHz}$. The phase bit in Fig. 4(b) was designed with $+270^{\circ}$ at $3.4 \mathrm{GHz}$ and $-270^{\circ}$ at $3.7 \mathrm{GHz}$ (PS270). The phase bit can be controlled by reconfiguring the transmission line length as shown in Fig. 4(c). To achieve constant phase steps of $90^{\circ}$ and $120^{\circ}$ for each antenna element, the

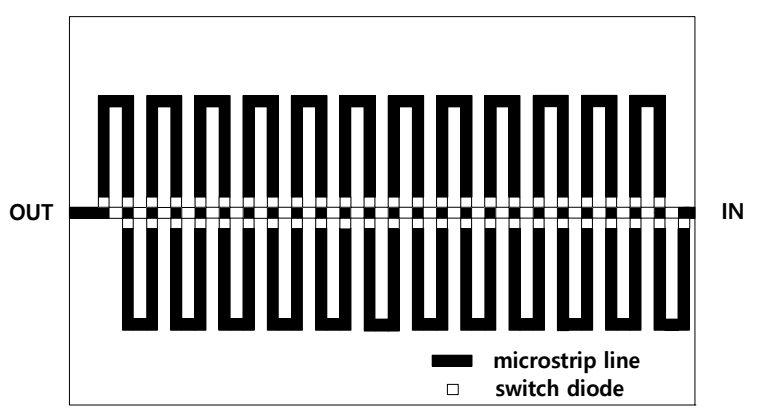

Fig. 3. Layout of the frequency-selective phase shifter with reconfigurable phase slopes.

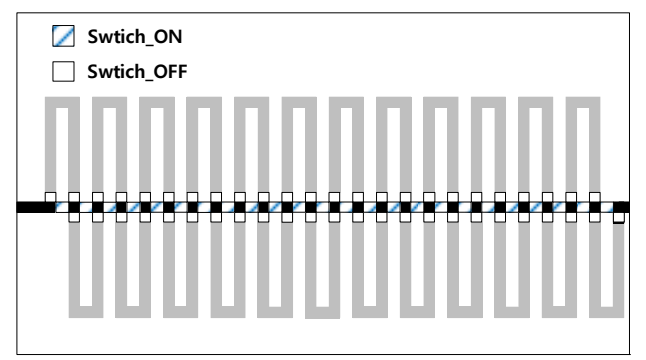

(a)

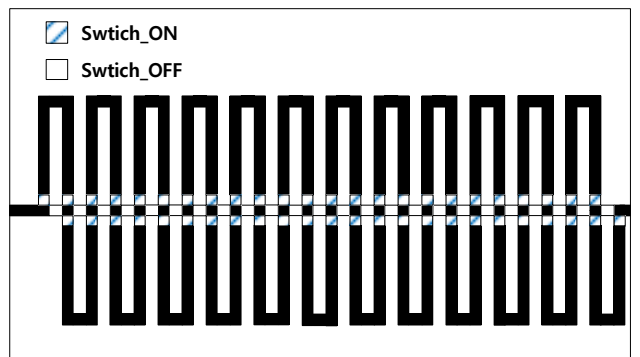

(b)

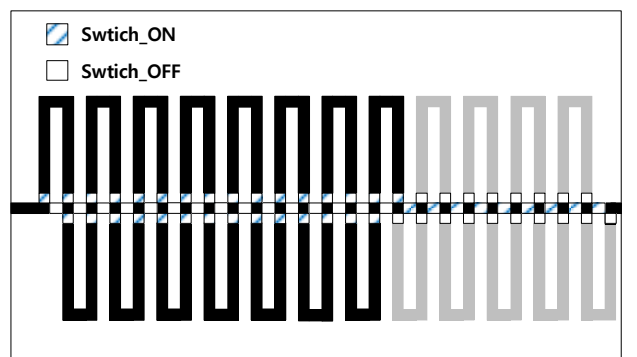

(c)

Fig. 4. Frequency-dependent phase shifter operations over 3.4 $\mathrm{GHz}$ to $3.7 \mathrm{GHz}$ with reconfigurable phase slopes of (a) $0^{\circ}$ (PS0), (b) $\pm 270^{\circ}$ (PS270), and (c) $\pm 180^{\circ}$ (PS180).

frequency-selective phase shifter was designed with six phase bits of PS0, PS90, PS120, PS180, PS240, and PS270.

Fig. 5 presents the phase shifts of PS0, PS90, PS120, PS180, PS240, and PS270. Since ideal PS0 should be not involve phase shift over the frequency band, the PS90 represents a relative

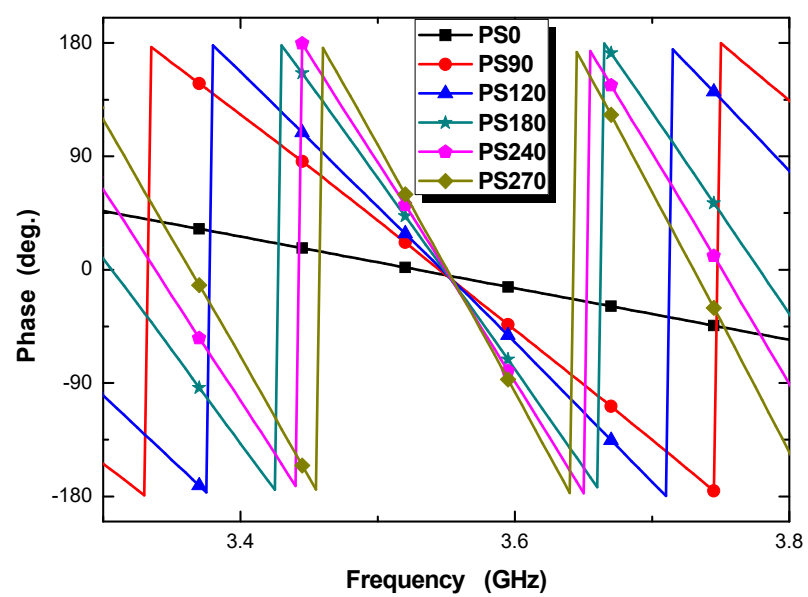

Fig. 5. Phase characteristics of the frequency-dependent phase shifter with reconfigurable phase slopes. 
phase shift referred to the phase in PS0 in which the phase varies from $+90^{\circ}$ to $-90^{\circ}$ within the operational frequency bands. Therefore, as each frequency channel has a different phase shift at each phase bit, the antenna beams steer one another directions.

\section{Planar Dual-Finger Wideband Antenna Element Design}

A single element antenna for the $5 \mathrm{G}$ planar array requires wideband design technology. A planar wideband antenna was designed with a dual-finger monopole configuration, as seen in Fig. 6(a). The microstrip feedline is transited to the planar monopole, and the antenna element has dimensions of $W=50$ $\mathrm{mm}, L=53 \mathrm{~mm}, a=2 \mathrm{~mm}, b=22 \mathrm{~mm}, c=20.12 \mathrm{~mm}, d=1$ $\mathrm{mm}, e=17 \mathrm{~mm}, g=1.88 \mathrm{~mm}$. The simulated and measured return losses are shown in Fig. 6(b). Due to the ultra-wideband design, the antenna element has a bandwidth of more than 2 $\mathrm{GHz}$. Moreover, the measured radiation pattern presents general omni-directional monopole patterns.

\section{Eight-Way Wideband Matched Feedline Design}

For the eight-element array, an 8-way feedline was designed to effectively distribute and transmit signal. The distance between the elements was determined to be $0.807 \lambda_{\mathrm{g}}$ of $44 \mathrm{~mm}$

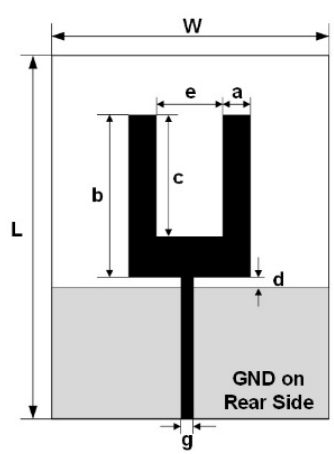

(a)

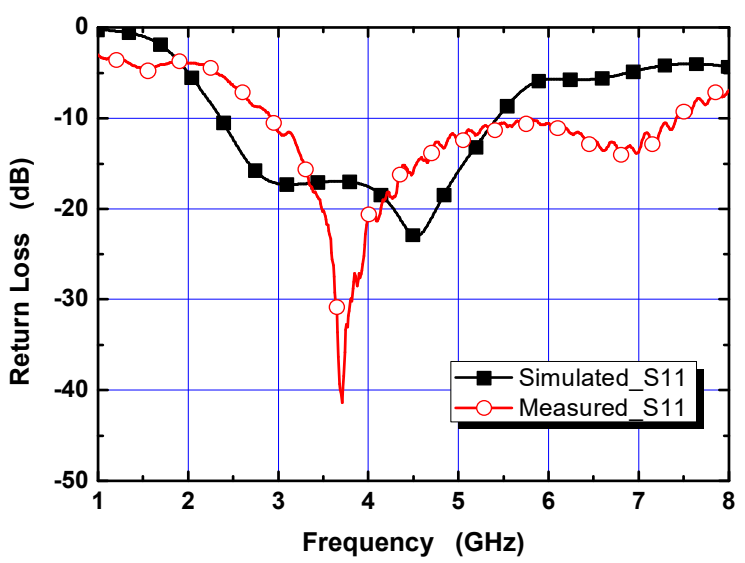

(b)

Fig. 6. Wideband antenna element: (a) layout and (b) return loss.

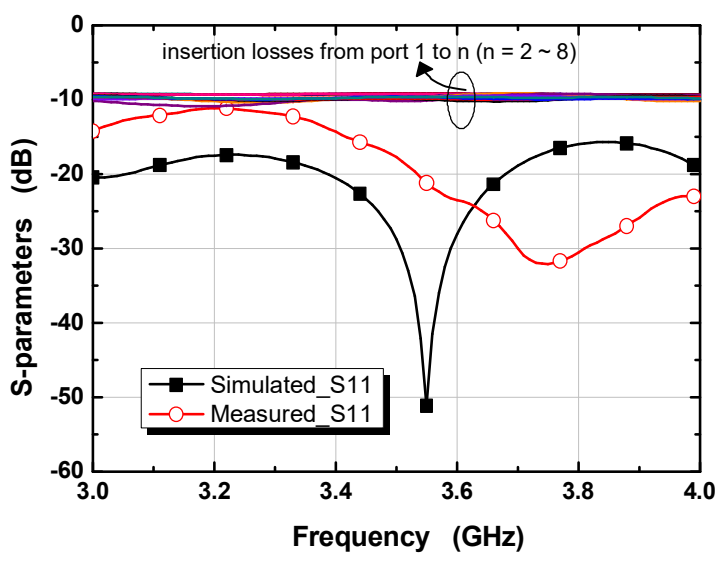

Fig. 7. Simulated and measured results of the 8-way feedline.

when considering the steered radiating beam patterns. The feedline was designed with tapered lines to match $50 \Omega$ to 100 $\Omega$ for the wideband operational frequency band. The three stage 2 -way divider was designed with tapered linewidths from 0.576 $\mathrm{mm}$ to $1.88 \mathrm{~mm}$. The simulated and measured return losses and insertion losses are shown in Fig. 7. A total of $10 \mathrm{~dB}$ insertion loss configuring a power division of $3 \mathrm{~dB} \times 3$ stages $=9 \mathrm{~dB}$ and implementation loss of about $1 \mathrm{~dB}$ was measured for each output port. Return loss was more than $10 \mathrm{~dB}$ at the specified $5 \mathrm{G}$ band. The 8-way tapered feedline was implemented with a size of $358 \times 92 \mathrm{~mm}^{2}$.

\section{IMPLEMENTATION AND PERFORMANCE EVALUATIONS}

The proposed frequency-selective beamforming array antenna system was implemented on an RF-301 substrate with a dielectric constant of 2.97 and a thickness of $0.762 \mathrm{~mm}$. The beamforming system layout is shown in Fig. 8. The array system consists of $8 \times 1$ dual-finger wideband monopole elements, frequency-selective phase shifters, and an 8-way wideband matched feedline. A ground plane is located on the rear side

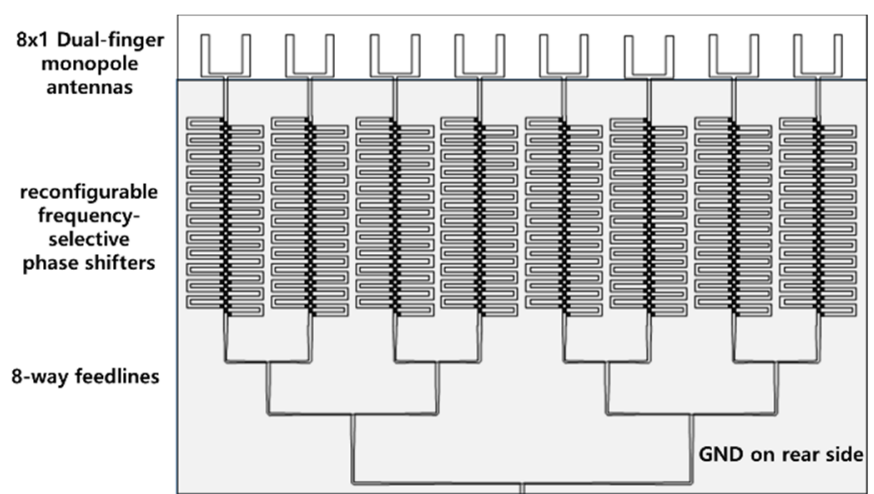

Fig. 8. Layout of the frequency-selective beamforming array antenna system. 
Table 1. Phase shifter setup for the experiments

\begin{tabular}{|c|c|c|c|c|c|c|c|c|}
\hline & \multicolumn{8}{|c|}{ Element No. } \\
\hline & 1 & 2 & 3 & 4 & 5 & 6 & 7 & 8 \\
\hline Fig. 9(a) & PS0 & PSO & PSO & PSO & PS0 & PS0 & PS0 & PS0 \\
\hline Fig. 9(b) & PS120 & PS0 & PS240 & PS120 & PS0 & PS240 & PS120 & PS0 \\
\hline Fig. 9(c) & PS0 & PS120 & PS240 & PS0 & PS120 & PS240 & PS0 & PS120 \\
\hline
\end{tabular}

except for the monopole antenna area. The overall size of the implemented system is $6.56 \lambda_{\mathrm{g}} \times 4.84 \lambda_{\mathrm{g}}$ of $358 \times 264 \mathrm{~mm}^{2}$.

The radiation patterns of the implemented system were measured in an anechoic chamber. For the radiation pattern measurements of the steered beams, the antenna elements were excited with the specified phase shifts as presented in Table 1. Fig. 9(a) shows the same direction beams for each frequency channel, while the PS0 is set to all phase shifters. Fig. 9(b) shows the radiation pattern with phase shifts for elements with a $-120^{\circ}$ phase step at $3.4 \mathrm{GHz}$ and $+120^{\circ}$ at $3.7 \mathrm{GHz}$, while patterns of the opposite phase set are presented in Fig. 9(c). Each beam at each frequency channel has a different beam steering angle. While Fig. 9(b) has beam steering angles of $-42^{\circ}$ at $3.4 \mathrm{GHz}, 0^{\circ}$ at $3.55 \mathrm{GHz}$ and $+38^{\circ}$ at $3.7 \mathrm{GHz}$, respectively, Fig. 9 (c) presents $+40^{\circ}$ at $3.4 \mathrm{GHz}, 0^{\circ}$ at $3.55 \mathrm{GHz}$ and $-38^{\circ}$ at $3.7 \mathrm{GHz}$, respectively. The performance of each beam pattern is summarized in Table 2. Overall, the experimental results exhibit the expected frequency-selective radiation beam steering and radiation performances.

\section{CONCLUSION}

In this paper, a frequency-selective beamforming phased array antenna system was proposed. While a conventional phased array presents only one directional beams for each frequency channel, the proposed channel-selective phased array can simultaneously radiate different beam directions at each channel using only $\mathrm{RF} / \mathrm{analog}$ processing, instead of complicated digital beamforming. In terms of its application to $5 \mathrm{G}$ mobile systems, a dual-finger shape planar monopole element with wide bandwidth characteristics was designed. The phase control circuit was designed with reconfigurable meander type phase shifters

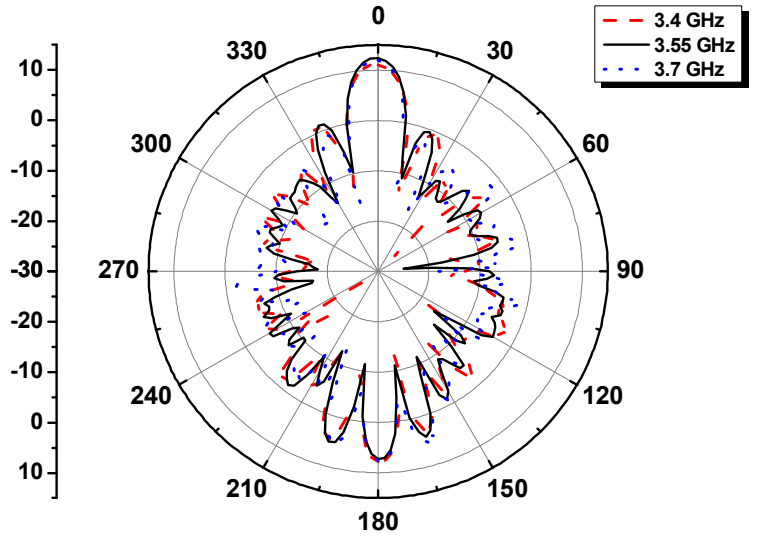

(a)

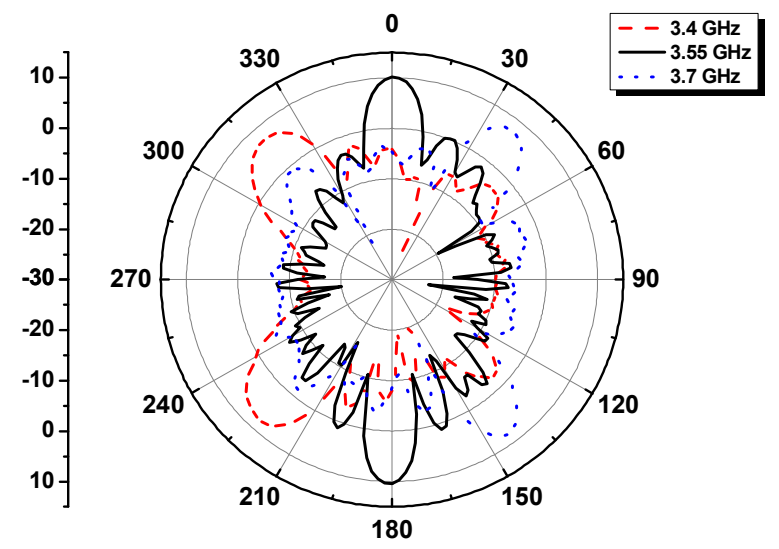

(b)

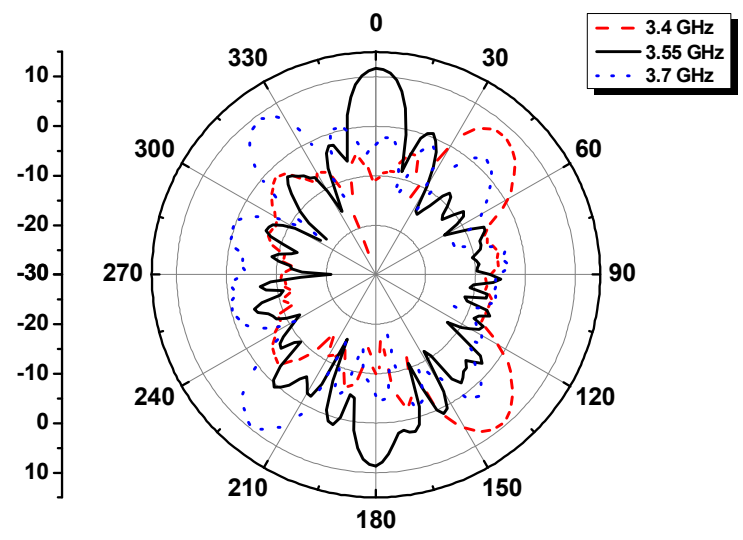

(c)

Fig. 9. Measured radiation patterns of the proposed frequencyselective phased array antenna system: (a) $0^{\circ}$, (b) forward beam, and (c) backward beam.

Table 2. Measured beam performances for each frequency-dependent beam

\begin{tabular}{|c|c|c|c|c|c|c|c|c|c|}
\hline & \multicolumn{3}{|c|}{ Fig. 9(a) } & \multicolumn{3}{|c|}{ Fig. 9(b) } & \multicolumn{3}{|c|}{ Fig. 9(c) } \\
\hline & $3.4 \mathrm{GHz}$ & $3.55 \mathrm{GHz}$ & $3.7 \mathrm{GHz}$ & $3.4 \mathrm{GHz}$ & $3.55 \mathrm{GHz}$ & $3.7 \mathrm{GHz}$ & $3.4 \mathrm{GHz}$ & $3.55 \mathrm{GHz}$ & $3.7 \mathrm{GHz}$ \\
\hline Steering angle $\left(^{\circ}\right)$ & 0 & 0 & 0 & -42 & 0 & +38 & +40 & 0 & -38 \\
\hline Gain $(\mathrm{dBi})$ & 10.96 & 12.32 & 11.87 & 7.83 & 10.4 & 7.99 & 9.32 & 11.63 & 9.45 \\
\hline Sidelobe level (dB) & -10.2 & -11.1 & -13.1 & -10.1 & -10.2 & -11.1 & -12.1 & -10.9 & -9.3 \\
\hline $\operatorname{HPBW}\left(^{\circ}\right)$ & 16 & 16 & 16 & 24 & 15 & 18 & 23 & 17 & 17 \\
\hline
\end{tabular}


that have various phase slopes within the wideband frequency range for narrow channel bandwidths. An 8-way feedline was designed as a tapered line with wideband characteristics to satisfy the $5 \mathrm{G}$ sub- $6 \mathrm{GHz}$ bands. From the experimental evaluation, the proposed array antenna system provided beam steering within the range of about $\pm 40^{\circ}$. As the beam steering angle varies from $0^{\circ}$ in both directions at each channel, inter-beam hand-off can be achieved in a small cell for $5 \mathrm{G}$ cellular applications, even though the proposed architecture of linear analog phase shifts provides frequency-dependent beam directions, unlike the digital beamforming system. The proposed frequency-dependent beamforming system can be considered a promising solution to achieve an adaptive, efficient beam control system for $5 \mathrm{G}$ mobile communication systems. Furthermore, wideband frequency-dependent phase shifters without time-delays can improve the system performance for wideband channel applications.

This The authors would like to appreciate for the APMC 2018 travel grant from the KIEES. This research was supported by the Basic Science Research Program through the NRF of Korea funded by the Ministry of Education (No. NRF-2015R1D1A3A01016567) and the Ministry of Science and ICT, Korea, under the ITRC support program supervised by the IITP (No. IITP-2019-2015-0-00403).

\section{REFERENCES}

[1] M. Shafi, A. F. Molisch, P. J. Smith, T. Haustein, P. Zhu, P. De Silva, F. Tufvesson, A. Benjebbour, and G. Wunder, "5G: a tutorial overview of standards, trials, challenges, deployment, and practice," IEEE Journal on Selected Areas in Communications, vol. 35, no. 6, pp. 1201-1221, 2017.

[2] S. J. Lee, W. S. Yoon, and S. M. Han, "Planar directional beam antenna design for beam switching system applications," Journal of Electromagnetic Engineering and Science, vol. 17, no. 1, pp. 14-19, 2017.

\section{Jung Ouk Kim}

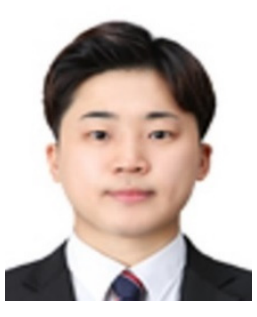

received the B.S. and M.S. degrees in information and communication engineering from Soonchunhyang University, Asan, Korea, in 2017 and 2019, respectively. He is currently a Research Engineer with the Military Division, RFHIC Corporation, Anyang, Korea. His current research interests include $\mathrm{RF} /$ microwave front-end systems, radar systems, and microwave components.
[3] S. Zihir and G. M. Rebeiz, "A 60 GHz 64-element phasedarray beam-pointing communication system for 5G 100 meter links up to $2 \mathrm{Gbps,"} \mathrm{in} \mathrm{Proceedings} \mathrm{of} 2016$ IEEE MTT-S International Microwave Symposium (IMS), San Francisco, CA, 2016, pp. 1-3.

[4] Y. Cao, K. S. Chin, W. Che, W. Yang, and E. S. Li, "A compact $38 \mathrm{GHz}$ multibeam antenna array with multifolded butler matrix for 5G applications," IEEE Antennas and Wireless Propagation Letters, vol. 16, pp. 2996-2999, 2017.

[5] J. Helander, K. Zhao, Z. Ying, and D. Sjoberg, "Performance analysis of millimeter-wave phased array antennas in cellular handsets," IEEE Antennas and Wireless Propagation Letters, vol. 15, pp. 504-507, 2015.

[6] S. H. Talisa, K. W. O'Haver, T. M. Comberiate, M. D. Sharp, and O. F. Somerlock, "Benefits of digital phased array radars," Proceedings of the IEEE, vol. 104, no. 3, pp. 530543, 2016.

[7] R. Mishra, R. G. Mishra, and P. Kuchhal, "Analytical study on the effect of dimension and position of slot for the designing of ultra wide band (UWB) microstrip antenna," in Proceedings of 2016 International Conference on Advances in Computing, Communications and Informatics (ICACCI), 2016, Jaipur, India, pp. 488-493.

[8] B. Kasi and C. K. Chakrabarty, "Ultra-wideband antenna array design for target detection," Progress in Electromagnetics Research, vol. 25, pp. 67-79, 2012.

[9] R. Garg and A. S. Natarajan, "A 28-GHz low-power phased-array receiver front-end with $360^{\circ}$ RTPS phase shift range," IEEE Transactions on Microwave Theory and Techniques, vol. 65, no. 11, pp. 4703-4714, 2017.

[10] D. M. Klymyshyn, S. Kumar, and A. Mohammadi, "Linear reflection phase shifter with optimised varactor gamma," Electronics Letters, vol. 33, no. 12, pp. 1054-1055, 1997.

[11] S. M. Han, K. H. Kwon, and Y. S. Kim, "New compact broadband phase shifters using coupled lines," Microwave and Optical Technology Letters, vol. 36, no. 5, pp. 399-400, 2003.

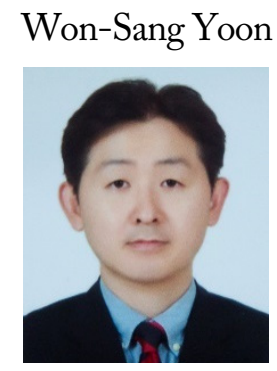

received the B.S., M.S., and Ph.D. degrees in radio sciences and engineering from Korea University, Seoul, Korea, in 1997, 1999, and 2010, respectively. From 1999 to 2015, he was a Senior Research Engineer with the Communication Research Center, Hanwha Systems Ltd., Yongin, Korea. Since 2015, he has been an Associate Professor with the Division of Electronics and Display Engineering, Hoseo University, Asan, Korea. His current research interests include RF/microwave front-end systems, reconfigurable antennas, and microwave components. 
Sang-Min Han

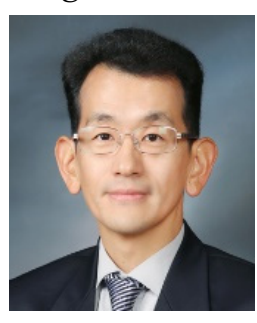

received the B.S., M.S., and Ph.D. degrees in radio sciences and engineering from Korea University, Seoul, Korea, in 1996, 1998, and 2003, respectively. From 1999 to 2001, he was a lecturer with the School of Electrical Engineering, Korea University. From 2003 to 2004, he was a Post-Doctoral Research Fellow with the University of California at Los Angeles (UCLA), USA. From 2005 to 2007, he was a Senior Research Engineer with the Samsung Advanced Institute of Technology (SAIT), Korea. In 2007, he joined Soonchunhyang University, Korea where he is currently a Professor with the Department of Information and Communication Engineering. He was a Visiting Research Faculty with Georgia Institute of Technology (GT), Atlanta, GA, USA from 2013 to 2014. Prof. Han is an Associate Editor of the Journal of the Korean Institute of Information Technology (JKIIT) and an Editorial Board Member of the Journal of Engineering (JE) and the Journal of Electromagnetic Engineering and Science (JEES), and a Senior Member of the IEEE. He appears in Marquis' Who's Who in Science and Engineering (2006-2007), Marquis' Who's Who in Asia (2007), and Marquis' Who's Who in the World (2018). His research interests include low-power RF/microwave system architectures for Smart Dust applications, spectrum sensing, active integrated antennas, and reconfigurable RF systems and devices. 